\title{
Performance of Steady-State Dispersion Models Under Low Wind-Speed Conditions
}

\author{
Wenjun Qian • Akula Venkatram
}

Received: 19 May 2010 / Accepted: 12 November 2010 / Published online: 3 December 2010

(C) The Author(s) 2010. This article is published with open access at Springerlink.com

\begin{abstract}
We examine the performance of two steady-state models, a numerical solution of the advection-diffusion equation and the Gaussian plume-model-based AERMOD (the American Meteorological Society/Environmental Protection Agency Regulatory Model), to predict dispersion for surface releases under low wind-speed conditions. A comparison of model estimates with observations from two tracer studies, the Prairie Grass experiment and the Idaho Falls experiment indicates that about 50\% of the concentration estimates are within a factor of two of the observations, but the scatter is large: the $95 \%$ confidence interval of the ratio of the observed to estimated concentrations is about 4 . The model based on the numerical solution of the diffusion equation in combination with the model of Eckman (1994, Atmos Environ 28:265-272) for horizontal spread performs better than AERMOD in explaining the observations. Accounting for meandering of the wind reduces some of the overestimation of concentrations at low wind speeds. The results deteriorate when routine one-level observations are used to construct model inputs. An empirical modification to the similarity estimate of the surface friction velocity reduces the underestimation at low wind speeds.
\end{abstract}

Keywords AERMOD · Low wind speed · Steady-state dispersion models · Wind meandering

\section{Introduction}

It is generally believed that commonly used steady-state Gaussian dispersion models, such as AERMOD (Cimorelli et al. 2005) are not applicable to situations when the wind speeds close to the ground are comparable to the standard deviation of horizontal velocity fluctuations. Under these conditions, the time scale of wind meandering is large compared to the

W. Qian $(\varangle) \cdot$ A. Venkatram

Mechanical Engineering, University of California, Riverside, A343 Bourns Hall, 900 University Ave., Riverside, CA 92507, USA

e-mail: windqian@gmail.com 
usual averaging time of $1 \mathrm{~h}$, and consequently the horizontal concentration distribution is far from Gaussian. Furthermore, routinely available mean wind measurements do not provide information on the turbulence levels required for modelling dispersion.

Other modelling approaches are considered more appropriate under these conditions. For example, Lagrangian particle models have been used to simulate dispersion under low windspeed conditions by Brusasca et al. (1992), Oettl et al. (2001), and Anfossi et al. (2006). The trajectories of these particles are governed by measured wind speed and turbulence levels as a function of time. Another approach (Arya 1995; Sharan et al. 1995; Sharan and Yadav 1998 ) is based on modifying the three-dimensional diffusion equation to include along-wind diffusion, which becomes important for low wind speeds. Venkatram et al. (2004) showed that a steady-state model can describe the concentration patterns under low wind speeds if it accounts for the directional distribution of the horizontal wind speed. The main message from all of these studies is that realistic estimates of concentrations under low wind-speed conditions can be made if meteorological measurements are made at time intervals that are much shorter than the averaging time used for the concentration. In most applications, such highly resolved measurements are not available and it is necessary to work with routine measurements resolved at 1-h intervals. This motivates the two questions addressed in our study: (1) How do steady-state dispersion models perform under low wind speeds, and (2) Can we use routine meteorological measurements, such as wind speed at one or several levels, to derive inputs required by dispersion models? We only address dispersion models applicable to source-receptor distances of a few kilometres. We also focus on surface releases under stable conditions when the surface wind speeds are typically low, and the concentration estimates from dispersion models can be relatively high.

To answer the first question, we consider two dispersion models. The first model is based on the numerical solution of the two-dimensional advection-diffusion equation, combined with the formulation for the horizontal plume spread proposed by Eckman (1994). This numerical solution provides an excellent description of surface-layer dispersion (e.g. van Ulden 1978), and represents the best available steady-state model. The performance of this model is compared with that of AERMOD (Cimorelli et al. 2005), the regulatory model recommended by the U.S. Environmental Protection Agency, which represents the current generation of dispersion models used in regulatory applications. The next section provides relevant details of these two models.

Cirillo and Poli (1992) and Sharan and Yadav (1998) have conducted studies similar to that described here, and we extend their results in three ways:

1. We evaluate models that have already been shown to perform well in describing groundlevel concentrations associated with surface releases during the moderate wind-speed conditions of the Prairie Grass experiment (Barad 1958).

2. We incorporate and examine the effects of the variation of wind speed with height on vertical and horizontal dispersion in the surface layer.

3. We propose and test methods to estimate the micrometeorological inputs required by these models under the low wind-speed conditions when Monin-Obukhov similarity is unreliable.

\section{Description of Models}

AERMOD (Cimorelli et al. 2005) uses the following formulation to estimate the ground-level concentration from a surface release during stable conditions: 


$$
C(x, y)=\frac{Q}{\sqrt{2 \pi} \sigma_{z} U_{e}} H(x, y)\left[\exp \left(-\frac{\left(H_{s}-z\right)^{2}}{2 \sigma_{z}^{2}}\right)+\exp \left(-\frac{\left(-H_{s}-z\right)^{2}}{2 \sigma_{z}^{2}}\right)\right],
$$

where $H_{s}$ is the effective stack height, and $z$ is the receptor height. Under low wind speeds, horizontal meandering of the wind spreads the plume over large azimuth angles, which might lead to concentrations upwind relative to the vector-averaged wind direction. AERMOD (Cimorelli et al. 2005), and other currently used regulatory models (Atmospheric Dispersion Modelling System (ADMS), Carruthers et al. 1994), attempt to treat this situation by assuming that when the mean wind-speed is close to zero, the horizontal plume spread covers $360^{\circ}$. Then, the concentration is taken to be a weighted average of concentrations of two possible states: a random spread state and a plume state. In the random spread state, the release is allowed to spread radially in all horizontal directions. Then, the weighted horizontal distribution in Eq. 1 is written as:

$$
H(x, y)=f_{r} \frac{1}{2 \pi r}+\left(1-f_{r}\right) \frac{1}{\sqrt{2 \pi} \sigma_{y}} \exp \left(-\frac{y^{2}}{2 \sigma_{y}^{2}}\right),
$$

where the first term represents the random state in which the plume spread covers $2 \pi$ radians, and $r$ is the distance between the source and receptor. The second term is the plume state corresponding to the Gaussian distribution. The plume is transported at an effective velocity given by

$$
U_{e}=\left(\sigma_{u}^{2}+\sigma_{v}^{2}+U^{2}\right)^{1 / 2}=\left(2 \sigma_{v}^{2}+U^{2}\right)^{1 / 2},
$$

where $U$ is the mean vector velocity, and the expression assumes that $\sigma_{v} \approx \sigma_{u}$. Note that the effective velocity is non-zero even when the mean velocity is zero. The minimum value of the transport wind, $U_{e}$, is $\sqrt{2} \sigma_{v}$. The weight for the random component in Eq. 2 is taken to be

$$
f_{r}=\frac{2 \sigma_{v}^{2}}{U_{e}^{2}}
$$

which ensures that the weight for the random component goes to unity when the mean wind approaches zero. ADMS uses a weighting scheme based on the mean wind speed. The success of this meandering correction in AERMOD depends on measurements of $\sigma_{v}$, which presumably reflect meandering when the wind speed is close to zero. If measurements are not available, we have to estimate $\sigma_{v}$ from other meteorological variables. The lateral dispersion, $\sigma_{y}$, in Eq. 1 is calculated from

$$
\sigma_{y}=\frac{\sigma_{v} x}{U_{e}(1+\alpha X)^{p}},
$$

where $X\left(=\sigma_{v} x / U_{e} z_{i}\right)$ is the non-dimensional distance defined in terms of the effective wind speed, $U_{e}$, and standard deviation of horizontal turbulent velocity component, $\sigma_{v}$. This formulation for $X$, incorporated in AERMOD, assumes that the Lagrangian time scale for lateral spreading is governed by eddies comparable to the height, $z_{i}$, of the boundary layer; the time scale is taken to be proportional to $z_{i} / \sigma_{v}$, During stable conditions, $z_{i}$ is estimated from $2300 u_{*}^{3 / 2}$ (Venkatram 1980), while $\alpha=78$ and $p=0.3$ are empirically determined values.

The vertical spread, $\sigma_{z}$, of a surface release is estimated from (Venkatram 1992)

$$
\sigma_{z}=\sqrt{\frac{2}{\pi}} \frac{u_{*} x}{U_{e}}\left(1+0.7 \frac{x}{L}\right)^{-1 / 3}
$$


where $L$ is the Obukhov length defined by $L=-T_{0} u_{*}^{3} /\left(\kappa g Q_{0}\right)$, where $Q_{0}$ is the surface kinematic heat flux, $u_{*}$ is the surface friction velocity, $g$ is the acceleration due to gravity, $T_{0}$ is a reference temperature, and $\kappa$ is the Von Karman constant taken to be 0.35 as in van Ulden (1978). Equation 6 is inferred from concentrations measured during the Prairie Grass experiment (Barad 1958). The surface friction velocity, $u_{*}$, is estimated from the wind speed measured at one level and an estimated roughness length, $z_{0}$. In the absence of measurements, $\sigma_{v}$ is taken to be $1.9 u_{*}$.

The second dispersion model is based on the numerical solution of the two-dimensional advection diffusion equation for crosswind integrated concentration, $\bar{C}^{y}$,

$$
U \frac{\partial \bar{C}^{y}}{\partial x}=\frac{\partial}{\partial z}\left(K \frac{\partial \bar{C}^{y}}{\partial z}\right),
$$

where horizontal diffusion is neglected. The profiles of wind speed $U$ and eddy diffusivity $K$ are given by Businger (1973). During stable conditions, the wind speed at height $z$ is given by

$$
U(z)=\frac{u_{*}}{\kappa}\left[\ln \left(\frac{z}{z_{0}}\right)+4.7 \frac{\left(z-z_{0}\right)}{L}\right]
$$

where $z_{0}$ is the aerodynamic roughness length. The effective distance, $z$, from the ground is obtained by subtracting the zero-plane displacement $\left(d_{h}\right)$ from the measurement height $\left(z_{r}\right)$. The eddy diffusivity, $K$, is taken to be equal to the diffusivity for heat:

$$
K=\kappa u_{*} z / \phi_{h},
$$

where

$$
\phi_{h}=0.74(1+6.3 z / L)
$$

during stable conditions. Equation 7 is solved numerically using the boundary condition

$$
\frac{\partial \bar{C}^{y}}{\partial z}=0
$$

at $z=0$ and $z=z_{i}$. When deposition is taken into account, the boundary condition at $z=0$ becomes

$$
K \frac{\partial \bar{C}^{y}}{\partial z}=v_{g} \bar{C}^{y},
$$

where $v_{g}$ is the deposition velocity close to the ground. The source strength is represented as

$$
\bar{C}^{y}\left(0, H_{s}\right)=\frac{Q}{U\left(H_{s}\right)} \delta\left(z-H_{s}\right) .
$$

Gryning et al. (1983) used a similar numerical approach to explain concentrations measured during the Prairie Grass experiment (Barad 1958). Surprisingly, there is little consensus on the calculation of horizontal plume spread for near-surface releases. Based on results from an earlier study (Venkatram 2004), we model the horizontal spread using Eckman (1994) hypothesis

$$
\frac{d \sigma_{y}}{d x}=\frac{\sigma_{v}}{\bar{U}}
$$


where the mean horizontal velocity of the plume, $\bar{U}$, is calculated from

$$
\bar{U} \equiv \int_{0}^{\infty} U \bar{C}^{y} d z / \int_{0}^{\infty} \bar{C}^{y} d z .
$$

Equation 12 can be integrated numerically to yield $\sigma_{y}$ as a function of downwind distance $x$. Notice that $\sigma_{y}$ grows rapidly close to the source where $\bar{U}$ is small and then slows down as the plume grows vertically into regions where the mean wind speed is larger.

Then, the centreline concentration is given by

$$
C(x, 0, z)=\frac{\bar{C}^{y}}{\sqrt{2 \pi} \sigma_{y}} .
$$

In order to apply these models, measurements of $u_{*}, \sigma_{v}$ and $L$ are needed as inputs. If measurements are not available, we have to make estimates of these variables from routine meteorological variables. We examine the performance of these models in explaining concentrations measured in two field studies, Prairie Grass (Barad 1958), and Idaho Falls (Sagendorf and Dickson 1974), when the surface wind speeds were relatively low.

\section{Field Studies}

\subsection{Prairie Grass Field Study}

The Prairie Grass Project (Barad 1958) provides a complete set of data for the analysis of surface-layer dispersion. The tracer, $\mathrm{SO}_{2}$, was released at a height of $0.46 \mathrm{~m}$, for an interval of $10 \mathrm{~min}$, and the concentration was sampled with five arcs at 50,100, 200, 400, and $800 \mathrm{~m}$ distance from the release. The samplers on the arcs were spaced at $2^{\circ}$ intervals on the first four arcs, and at $1^{\circ}$ on the $800-\mathrm{m}$ arc. Half of the 70 experiments were conducted during stable conditions, which covered both low and high wind-speed conditions. The data were obtained from http://www.dmu.dk/International/Air/Models/Background/ExcelPrairie.htm. Note that the friction velocity and the Obukhov length were not measured but obtained by fitting similarity profiles to the mean wind speed and temperature measured at several levels on a tower. We focus on cases when the wind speed was less than $2 \mathrm{~m} \mathrm{~s}^{-1}$ at the tower level of $1 \mathrm{~m}$.

\subsection{Idaho Falls Field Study}

The Idaho Falls experiment (Sagendorf and Dickson 1974), which focuses on low winds in stable conditions, was conducted at the Idaho National Engineering Laboratory (INEL) in a broad, relatively flat plain. $\mathrm{SF}_{6}$ was released at a height of $1.5 \mathrm{~m}$, and samplers were placed at intervals of $6^{\circ}$ on arcs of radii 100, 200, and $400 \mathrm{~m}$ from the release. The receptor height was $0.76 \mathrm{~m}$. Wind measurements were provided by lightweight cup anemometers and bivanes at heights of $2,4,8,16,32$, and $61 \mathrm{~m}$ on the $200-\mathrm{m}$ arc. We estimated $u_{*}$ and $L$ from the tower measurements, but this required an estimate of the aerodynamic roughness length, $z_{0}$. Brusasca et al. (1992) and Sharan and Yadav (1998) estimated $z_{0}$ to be $0.005 \mathrm{~m}$ for Idaho Falls, obtaining the value by fitting a neutral wind profile to observed winds at several levels for the only neutral case (Test 6) in the Idaho Falls experiment. This method might not be reliable because it is based on only one case. We recalculated $z_{0}$ using the data from all the tests. We calculated the optimum $z_{0}$ by minimizing the coefficient of variation of $u_{*}$ 
corresponding to the wind speeds measured at 2, 4, and $16 \mathrm{~m}$ (the 8-m wind measurement was questionable because it was often lower than that at $4 \mathrm{~m}$ ). The best estimate of $z_{0}$ turned out to be $0.08 \mathrm{~m}$.

\section{Model Performance}

The performance of the models considered here can be described using a variety of statistics, described in Chang and Hanna (2004). We have chosen to use the geometric mean $\left(m_{g}\right)$ and the standard deviation $\left(s_{g}\right)$ of the ratios of the observed to modelled variable as the primary measures of model performance because they can be readily interpreted (Venkatram 2008). They are defined as:

$$
\begin{aligned}
& m_{g}=\exp \left(\left\langle\varepsilon_{m}\right\rangle\right), \\
& s_{g}=\exp \left(\sigma\left(\varepsilon_{m}\right)\right),
\end{aligned}
$$

where \langle\rangle and $\sigma$ represent mean and standard deviation respectively, and $\varepsilon_{m}$ is the residual between the logarithms of the model estimate and observation,

$$
\varepsilon_{m}=\ln \left(C_{p}\right)-\ln \left(C_{o}\right)
$$

where $C_{o}$ and $C_{p}$ are observed values and corresponding estimates respectively. The angle brackets refer to an average. The deviation of the geometric mean, $m_{g}$, from unity indicates whether the model is underpredicting or overpredicting, and is a measure of bias of the model estimate. The geometric standard deviation, $s_{g}$, is a measure of the uncertainty in the model prediction with $s_{g}^{2}$ being approximately the $95 \%$ confidence interval for the ratio of $C_{p} / C_{o}$. The calculation of the geometric mean, $m_{g}$, and the geometric standard deviation, $s_{g}$, using Eq. 15 poses problems when the observation is close to zero and the corresponding model estimate is finite; the large logarithm of the ratio dominates the calculation. This is avoided by equating $m_{g}$ to the median of the ratio of the observed to predicted concentration ratio, and using the interquartile range of the ratios to estimate $s_{g}$. The statistics of model performance include the correlation between model estimates and observations, $r^{2}$, and the fraction of the estimates within a factor of two of the observations, fact 2 , in addition to $m_{g}$ and $s_{g}$.

We first examine the performance of the numerical model in estimating the normalized crosswind integrated concentration, $\bar{C}^{y} / Q$, for all the stable cases that occurred during the Prairie Grass experiment. This ensures that our results are consistent with those obtained in earlier studies (Nieuwstadt and van Ulden 1978; van Ulden 1978). We used a typical value of deposition velocity equal to $0.01 \mathrm{~m} \mathrm{~s}^{-1}$ in the numerical model for $\mathrm{SO}_{2}$ dispersion (Gryning et al. 1983). The meteorological inputs are taken from Table 2 of van Ulden (1978). Figure 1 shows that the performance of the numerical method is similar to that from earlier studies. The bias between the model estimates and observations is only $4 \%$ and the correlation between the two sets is excellent $\left(r^{2}=0.90\right)$.

The performance of the model in explaining centreline concentrations is examined by separating the experiments into two sets: a low-wind set corresponding to wind speeds of less than $2 \mathrm{~m} \mathrm{~s}^{-1}$ at $1 \mathrm{~m}$, and the high-wind set that includes the remainder of the cases. The calculation of the horizontal spread of the plume requires the standard deviation of horizontal velocity fluctuations, $\sigma_{v}$, which is based on $\sigma_{\theta}$ measured at $1 \mathrm{~m}$. We estimate $\sigma_{v}$ from $\sigma_{\theta}$ and $U$ using a semi-empirical formulation suggested in Venkatram et al. (2004),

$$
\sigma_{v}=\operatorname{atanh}\left(\frac{\sigma_{\theta}}{\sigma_{\theta \max }}\right) \sigma_{\theta \max } U,
$$


Fig. 1 Comparison of crosswind-integrated concentrations $\left(\bar{C}^{y} / Q\right)$ from the numerical model with corresponding observations during stable conditions of the Prairie Grass experiment
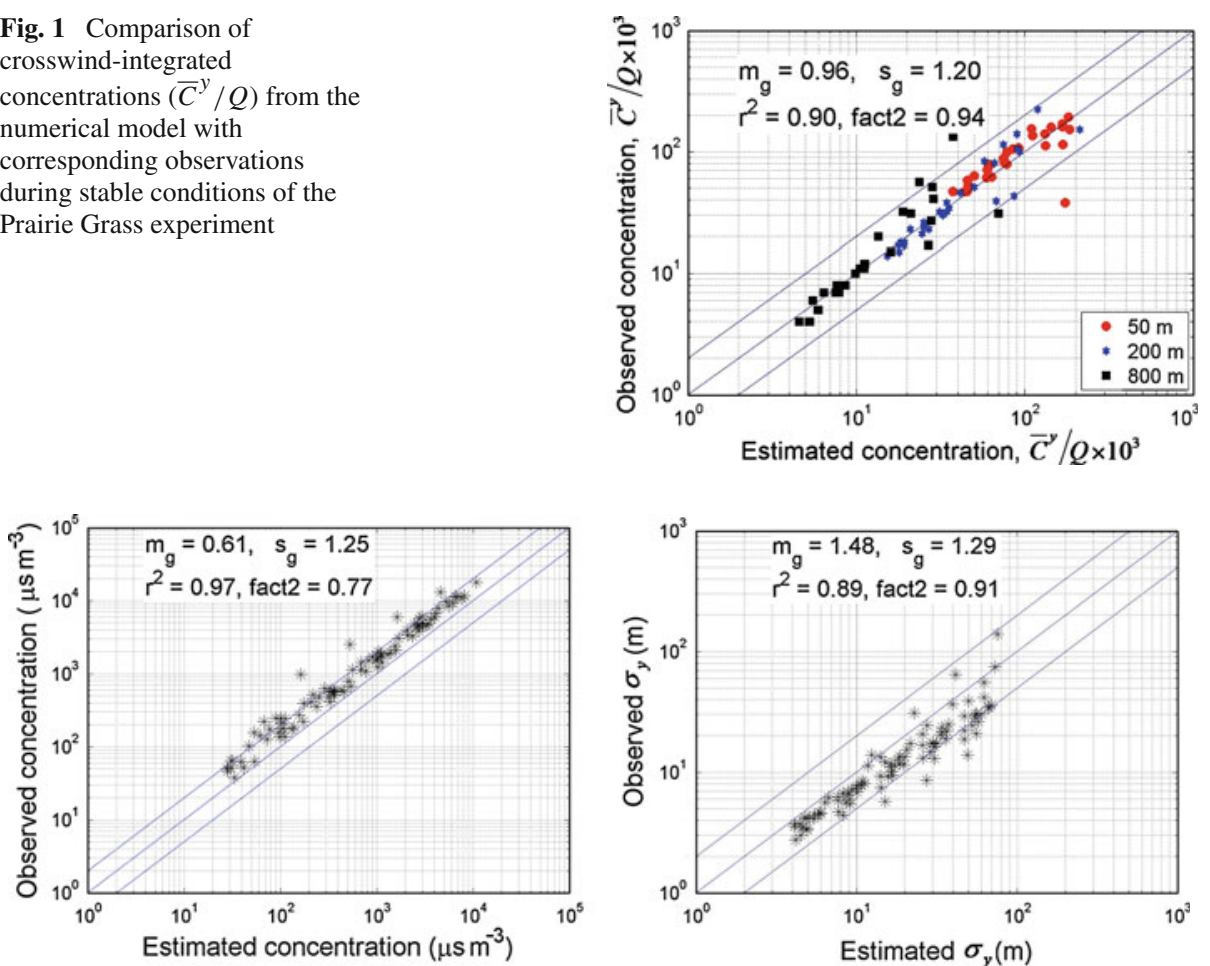

Fig. 2 Comparison of centreline concentrations $(C / Q)$ and horizontal plume spreads $\left(\sigma_{y}\right)$ from the numerical model with observations from the Prairie Grass experiment during high-wind stable conditions

where the maximum value of $\sigma_{\theta}$ is $\sigma_{\theta} \max =\pi / \sqrt{3}$, which corresponds to a uniform distribution of the wind direction over $2 \pi$. When $\sigma_{\theta}$ is small compared to $\sigma_{\theta \max }, \sigma_{v} \approx \sigma_{\theta} U$. Equation 17 yields results that are very close to those from a model suggested by Cirillo and Poli (1992), which assumes that the horizontal velocity fluctuations are determined by a constant magnitude wind vector whose direction is normally distributed. Luhar (2010) has recently extended this model by allowing the wind vector to vary in magnitude as well as direction. Luhar's expression is a viable alternative to Eq. 17 if measurements of the standard deviation of the scalar wind are available. The observed values of horizontal plume spread, $\sigma_{y}$, are obtained by fitting Gaussian distributions to observed concentrations at each arc.

Figure 2 shows that during high wind-speed conditions, the numerical method underestimates the observed concentrations by $39 \%$, and overestimates the horizontal plume spread, $\sigma_{y}$, by $48 \%$. But the estimates are well correlated with observations with $r^{2}$ larger than 0.89 . The underestimation of concentrations by the numerical method is clearly related to the overestimation of $\sigma_{y}$, which can be related to the use of a single value of $\sigma_{v}$ measured close to surface. For present purposes, we calibrated Eckman (1994) model with the observations by multiplying the right-hand side of Eq. 12 by a factor of 0.7 to reduce the value of $\sigma_{y}$. This results in the removal of most of the bias in the modelled concentration estimates.

This calibrated model is then used to explain the concentrations observed for the lowwind set. The upper panel of Fig. 3 shows that the calibrated numerical model overestimates the centreline concentrations by $42 \%$. The correlation coefficient between the estimates and observations is only 0.55 . The extreme overestimation for certain points correspond to the 

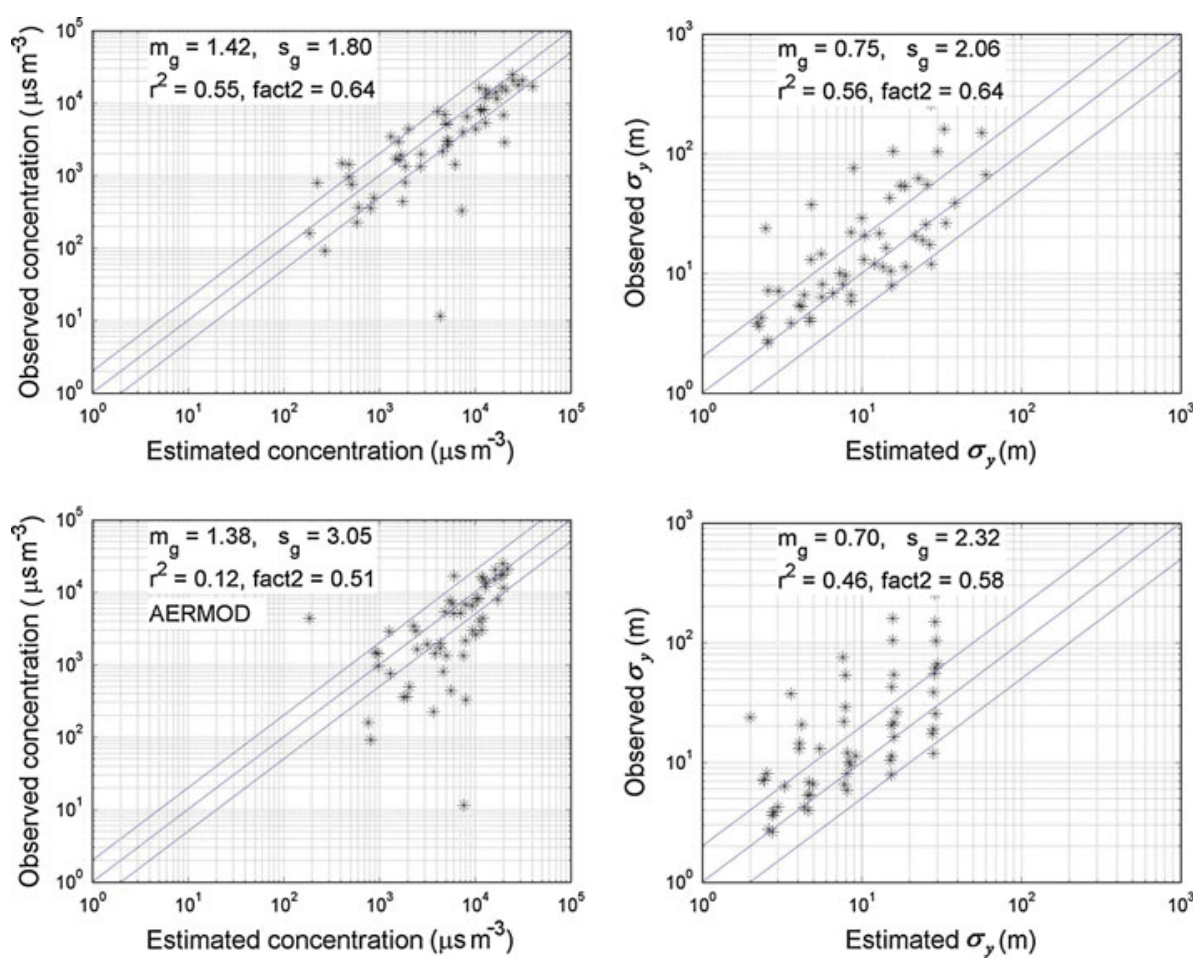

Fig. 3 Comparison of centreline concentrations $(C / Q)$ and horizontal plume spreads $\left(\sigma_{y}\right)$ from the numerical model (upper panel) and AERMOD (lower panel) with observations from the Prairie Grass experiment during low-wind stable conditions

lowest wind speed $\left(U=0.66 \mathrm{~m} \mathrm{~s}^{-1}\right)$ that occurred during the Prairie Grass experiment. The overestimation of concentrations is mainly due to the underestimation of $\sigma_{y}$, shown on the upper right plot of Fig. 3.

The performance of AERMOD is comparable to that of the numerical model when the wind speed is higher than $2 \mathrm{~m} \mathrm{~s}^{-1}$ at $1 \mathrm{~m}$. AERMOD's estimates of $\sigma_{y}$ are closer to observations because they are based on an empirical fit, Eq. 5, to the Prairie Grass data. The performance of AERMOD under low wind conditions is illustrated in Fig. 3. The lower panel shows that AERMOD overestimates the concentrations by $38 \%$ and underestimates $\sigma_{y}$ by $30 \%$ during low wind-speed conditions in the Prairie Grass experiment. The scatter in the concentration estimates $\left(s_{g}=3.05\right)$ is much larger than that of the numerical model $\left(s_{g}=1.80\right)$. The correlation between the concentration estimates and observations using $\operatorname{AERMOD}\left(r^{2}=0.12\right)$ is also smaller than that of the numerical model $\left(r^{2}=0.55\right)$.

These results show that the numerical steady-state model provides adequate estimates of concentrations and plume spreads because it has a justifiable description of the interaction between dispersion and the gradient of the wind speed near the surface. The meandering correction does not play a role for these cases because $\sigma_{v}$ is small compared to the mean wind speed at $1 \mathrm{~m}$. Note that Eckman's model describes horizontal plume spread even under low wind speeds as long as measured values of $\sigma_{v}$ are used. The horizontal plume spread relation in AERMOD does not perform as well as that based on Eckman's hypothesis. 
We next address the second question of the paper: how reliable are the estimates of the meteorological inputs when only routine observations at one level are available?

\section{Estimating Meteorological Inputs}

The meteorological inputs required by the models are the surface friction velocity, $u_{*}$, the Obukhov length, $L$, and the standard deviation of the horizontal velocity fluctuations, $\sigma_{v}$. We estimate these variables using the wind speed measured at one level, and an estimate of the surface roughness length, $z_{0}$.

The surface friction velocity is estimated using a method proposed in Venkatram (1980; Venkatram and Princevac 2008) and currently incorporated in AERMET, AERMOD's meteorological processor. It is based on Monin-Obukhov similarity theory for the profile of the mean wind, $U$ (see Eq. 8). Analysis of data from field experiments conducted in Kansas (Izumi 1971), Minnesota (Caughey et al. 1979) and Prairie Grass (Barad 1958) shows that the temperature scale, $T_{*}=-\overline{w^{\prime} T^{\prime}} / u_{*}$ varies little with $u_{*}$ and can be taken to be about $0.08 \mathrm{~K}$. Assuming a constant value of $T_{*}$ in Eq. 8 results in the following equation for $u_{*}$ :

$$
u_{*}=\frac{C_{D N}^{1 / 2} U}{2}\left[1+\left(1-r^{2}\right)^{1 / 2}\right]
$$

where $C_{D N}$ is the drag coefficient for neutral conditions,

$$
C_{D N}=\frac{\kappa^{2}}{\left(\ln \left(\frac{z_{r}-d_{h}}{z_{0}}\right)\right)^{2}},
$$

where $z_{r}$ is the measurement height, and $r$ is the ratio between the critical wind speed, $U_{c r i t}$, and measured wind speed, $U$

$$
r=\frac{U_{c r i t}}{U}
$$

with

$$
U_{c r i t}=\frac{2 u_{0}}{C_{D N}^{1 / 4}}
$$

and

$$
u_{0}=\left(\frac{\beta g\left(z_{r}-d_{h}-z_{0}\right) T_{*}}{T_{0}}\right)^{1 / 2} .
$$

Equation 18 does not have real solutions for $r>1$. Under such conditions, the surface friction velocity is computed as half of the neutral value,

$$
u_{*}=C_{D N}^{1 / 2} U / 2
$$

In our analysis, we approximate the offending term by

$$
\left(1-r^{2}\right)^{1 / 2} \simeq \exp \left(-\frac{r^{2}}{2}\right),
$$

and so the expression for $u_{*}$ becomes

$$
u_{*}=\frac{C_{D N}^{1 / 2} U}{2}\left[1+\exp \left(-\frac{r^{2}}{2}\right)\right] .
$$


The vertical turbulent velocity, $\sigma_{w}$, which is not used directly in the models, is proportional to the surface friction velocity (e.g. Panofsky and Dutton 1984) with $\sigma_{w}=1.3 u_{*}$, while the horizontal turbulent velocity, $\sigma_{v}$, is related to the friction velocity through $\sigma_{v}=1.9 u_{*}$. Assuming a constant temperature scale, $T_{*}$, this results in $L=1100 u_{*}^{2}$.

We first examine the performance of Eq. 25 and expressions for turbulent velocities with data from the Cardington experiment, which includes turbulence measurements during low wind-speed conditions.

\subsection{Cardington Experiment}

The data analysed here were collected at a meteorological tower operated by the UK Meteorological Office at Cardington, Bedfordshire (see http://badc.nerc.ac.uk/data/cardington/). The tower, located on a large grassy field, has sonic anemometers making wind and temperature measurements at 10, 25, and $50 \mathrm{~m}$ above the ground. These data are sampled at $50 \mathrm{~Hz}$, and the vector mean winds, temperatures, turbulent fluxes and variances are averaged over 1 , 10 , and $30 \mathrm{~min}$. We used the 30-min averages from 10-m height in our analysis. The dataset corresponds to all the stable periods $(L>0)$ for 2005 . The aerodynamic roughness length, $z_{0}=0.025 \mathrm{~m}$, was obtained by fitting the similarity wind-speed profile to observations during near-neutral conditions $(|L|>200 \mathrm{~m})$. This value is the same as that used by Luhar et al. (2009) in their study of low wind-speed conditions.

The left panel of Fig. 4 shows the variation of $u_{*} / u_{*_{n}}$ with $U / U_{c r i t}$, where $u_{*_{n}}$ is the friction velocity assuming neutral conditions. The dashed line representing Eq. 24 follows the variation when $U / U_{\text {crit }} \geq 1$, but when the wind speed approaches zero, the ratio of $u_{*} / u_{*_{n}}$ approaches values much larger than half of the neutral values. We propose the following tentative modification to Eq. 25 shown by the solid line to better follow the variation when the wind speed is low:

$$
u_{*}=\frac{C_{D N}^{1 / 2} U}{2} \frac{1+\exp \left(-r^{2} / 2\right)}{1-\exp (-2 / r)} \text {. }
$$

This modification leads to a limit for $u_{*}=C_{D N}^{1 / 2} U_{c r i t} / 4$ when the wind speed approaches zero. On the right panel of Fig. 4, we see that this limit lies in the middle of the measured values when the wind speed is close to zero, while Eq. 25 gives much lower $u_{*}$ estimates. The performance of Eq. 25 in estimating $u_{*}$ is compared to that of Eq. 26 in the left panel of Fig. 5. Estimates of $u_{*}$ from Eq. 25 (stars) are scattered when the observed $u_{*}$ is low. The modification of Eq. 26 (dots) reduces most of the underestimation in $u_{*}$. The bias between the estimates and observations is reduced from $7 \%$ to zero and the scatter is also reduced with $s_{g}$ decreasing from 1.31 to 1.27 . However, the proposed modification does not decrease the bias when $u_{*}$ is overestimated. The right panel of Fig. 5 shows that $\sigma_{v}$ is also underestimated if the linear relation, $\sigma_{v}=1.9 u_{*}$, is used and $u_{*}$ is estimated from Eq. 25. The modification of Eq. 26 removes most of the underestimation of $\sigma_{v}$, which can be seen from the reduction of bias from 16 to $9 \%$. However, a fraction of the values is still underestimated.

\section{Model Performance with Estimated Meteorological Parameters}

We first use the numerical model to estimate the concentrations, but the meteorological inputs are based on measurements at the 1-m level. The upper panels of Fig. 6 show estimates based on the observed values of $\sigma_{v}$, while the lower panels show concentrations based on values of 

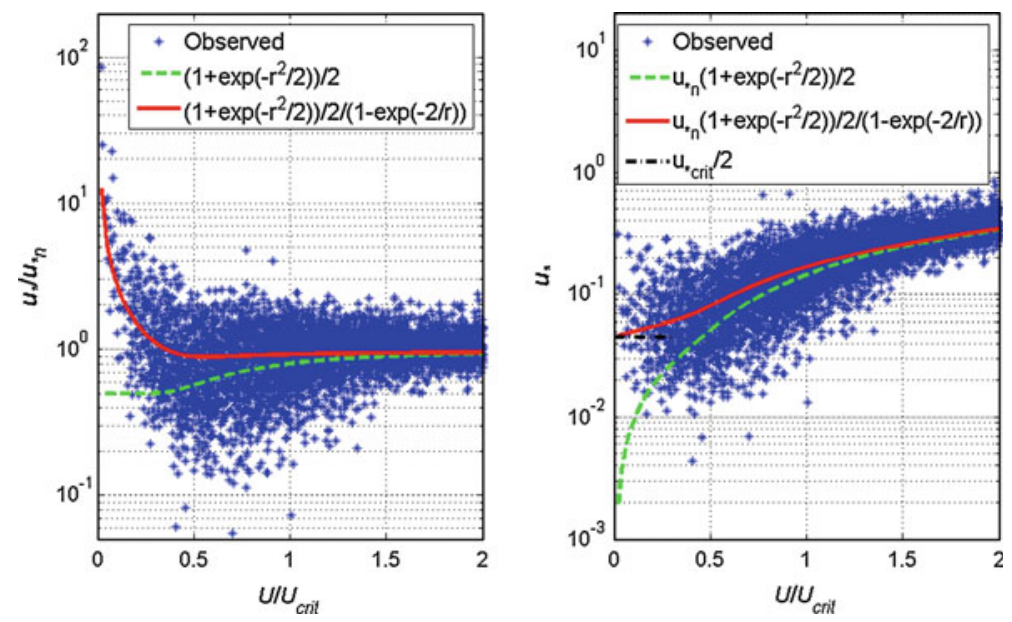

Fig. 4 Variation of $u_{*} / u_{*_{n}}$ (left) and $u_{*}$ (right) with $U / U_{\text {crit }}$ see Eq. 21. Stars correspond to observations, dash lines correspond to Eq. 25, solid lines correspond to Eq. 26, and the dash-dot line represents the limit
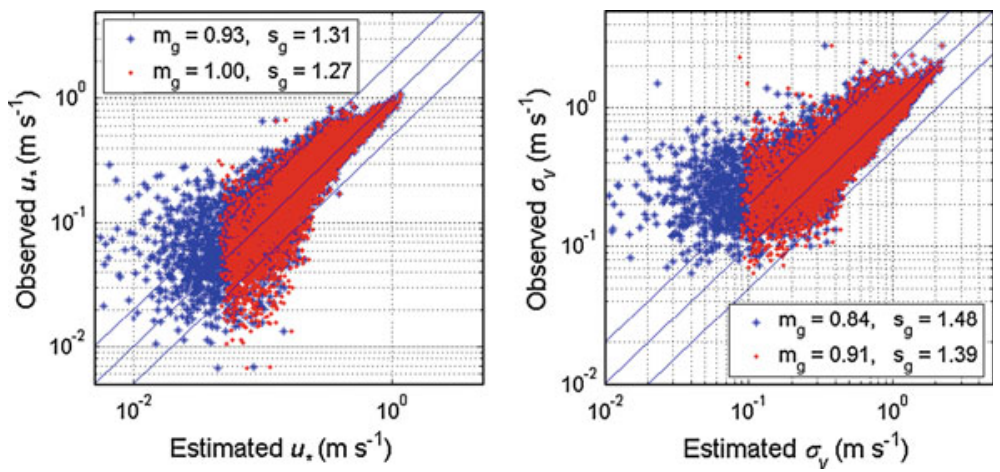

Fig. 5 Comparison of $u_{*}$ (left panel) and $\sigma_{v}$ (right panel) estimates with observations from the Cardington site. The stars correspond to Eq. 25 and dots correspond to Eq. 26. $\sigma_{v}$ is estimated from $\sigma_{v}=1.9 u_{*}$ using the $u_{*}$ estimates from the left panel

$\sigma_{v}$ related to $u_{*}$ using Eq. 26. The upper panel indicates that the bias between the concentration estimates and observations is reduced to $33 \%$, from $42 \%$, corresponding to the surface friction velocity estimated from similarity relations (see Fig. 3). The correlation between the estimates and observations is also better, $r^{2}=0.62$ here compared with $r^{2}=0.55$ in the upper panel of Fig. 3. The correlation between the estimated and observed $\sigma_{y}$ is also slightly increased $\left(r^{2}=0.58\right)$ compared with $r^{2}=0.56$ in the upper panel of Fig. 3, indicating that for this limited dataset, using friction velocities based on a single wind speed, yields results that are at least as good as those based on surface friction velocities derived from similarity relations.

However, the lower panel of Fig. 6 shows that, when $\sigma_{v}$ is estimated from the surface friction velocity, model performance deteriorates. The centreline concentration is underestimated by $37 \%$ while $\sigma_{y}$ is overestimated.

Figure 7 shows the performance of the numerical model for the Idaho Falls data, where the observed $\sigma_{\theta}$ is used to calculate $\sigma_{v}$ with Eq. 17. The friction velocity and Obukhov length 

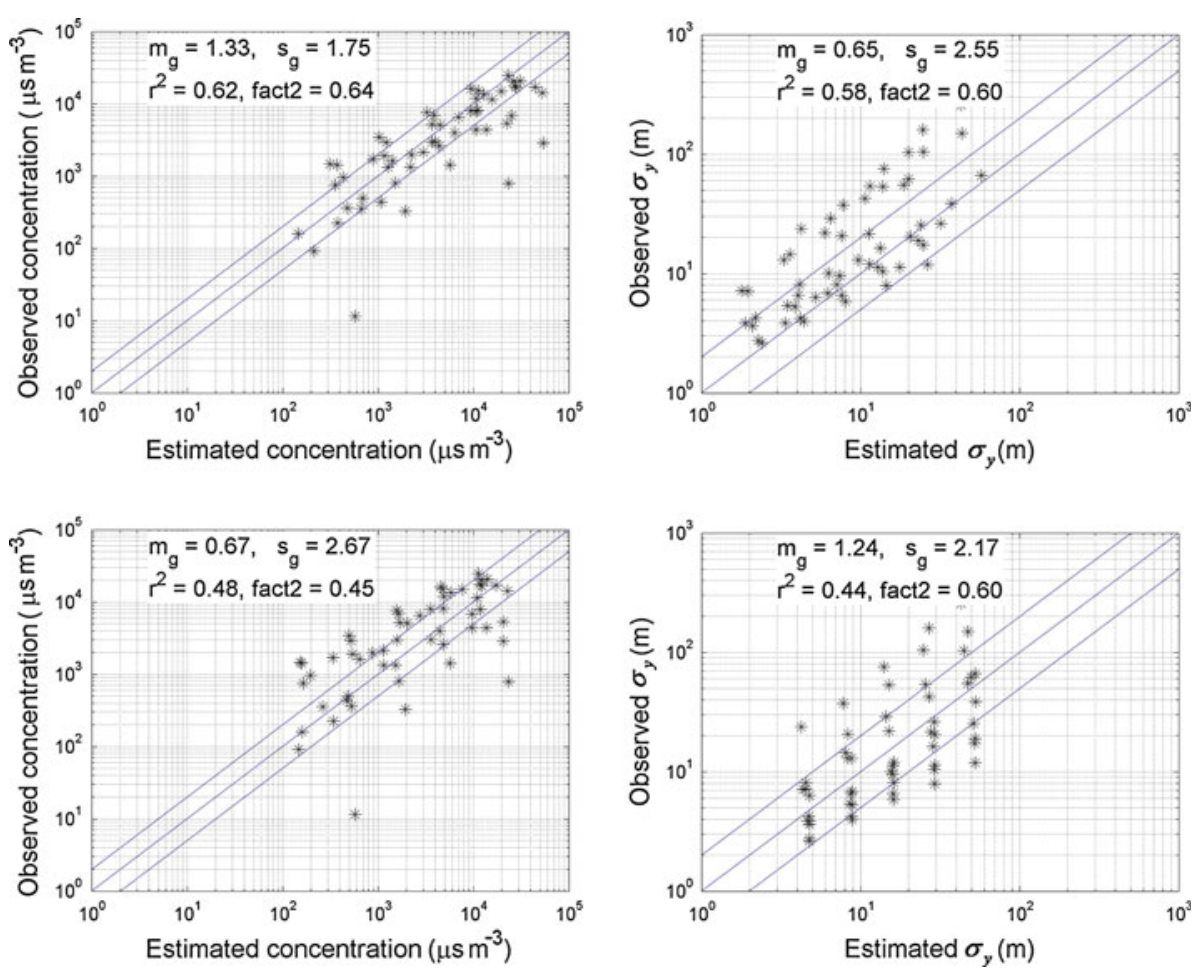

Fig. 6 Comparison of centreline concentrations $(C / Q)$ and horizontal plume spreads $\left(\sigma_{y}\right)$ from the numerical model with observations from the Prairie Grass experiment during low-wind stable conditions. $u_{*}$ and $L$ are estimated using Eq. 26. The upper panel uses observed $\sigma_{v}$, and the lower panel uses $\sigma_{v}=1.9 u_{*}$

are estimated using Eq. 26 and the approximation $L=1100 u_{*}^{2}$. The left panel shows that the centreline concentration is overestimated by $61 \%$ with the correlation coefficient between the estimates and observations, $r^{2}=0.48$. The overestimation of the centreline concentration can be reduced by including meandering into the formulation in AERMOD:

$$
C=\bar{C}^{y}\left[\frac{\left(1-f_{r}\right)}{\sqrt{2 \pi} \sigma_{y}}+\frac{f_{r}}{2 \pi r}\right] .
$$

The performance of the modified numerical model is shown on the right plot of Fig. 7. The overestimation of the concentration is reduced when meandering is included in the numerical model, with $m_{g}$ decreasing from 1.61 to 1.31 . But the concentration is still overestimated for some cases. Figure 7 compares the performance of the numerical method with that of AERMOD using the Idaho Falls experimental data. AERMOD overestimates concentrations by $72 \%$ even though meandering is included. The correlation coefficient between the estimates and observations is worse than that from the numerical method $\left(r^{2}=0.39\right.$ compared with $r^{2}=0.50$ in the upper right plot of Fig. 7).

The effect of meandering on the performance of the numerical model becomes apparent by examining the concentration distribution on the $50-\mathrm{m}$ arc. Concentration estimates from the numerical model with and without meandering are plotted against the observations as a function of the receptor angle relative to the wind direction. The left plot of Fig. 8 shows results for Test \# 10, when the wind speed is relatively high and the random fraction is 


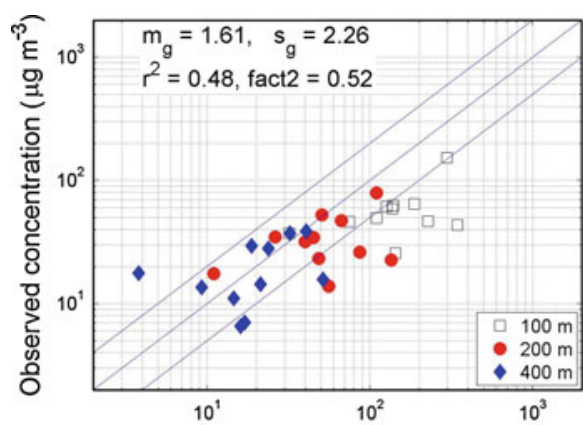

Estimated concentration $\left(\mu \mathrm{g} \mathrm{m}^{-3}\right)$



Estimated concentration $\left(\mu \mathrm{g} \mathrm{m}^{-3}\right)$

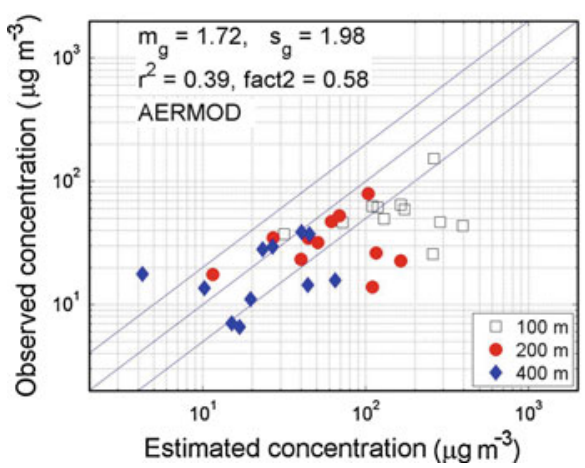

Fig. 7 Comparison of centreline concentrations from the numerical model (upper panel) and AERMOD (lower panel) with observations from the Idaho Falls experiment. Observed $\sigma_{\theta}$ is used to calculate $\sigma_{v}$. No meandering is considered in the upper left plot while meandering is considered in the upper right plot

relatively low. The observed data show two peaks of concentration, which cannot be described by the steady-state model. The maximum concentration estimated from the numerical model with no meandering is higher than the observed maximum. Accounting for meandering in the model brings the maximum concentration closer to the observed value.

The right plot of Fig. 8 shows results for Test \# 8, for the wind speed when the random fraction has its highest value. We see that the concentrations are observed at large azimuth angles relative to the wind direction, indicating wind meandering. The maximum concentration from the numerical model is much larger than the observed value even when meandering is considered. These results show that accounting for meandering reduces the overestimation of maximum concentrations during low wind-speed conditions. However, when meandering is large, estimated concentrations are still much larger than the observed values indicating that the actual vertical spread is much larger than that estimated using the surface friction velocity.

Figure 9 shows that setting $\sigma_{v}=1.9 u_{*}$ results in overestimation of concentrations because $\sigma_{y}$ is underestimated. The meandering component does not help because of the underestimation of $\sigma_{v}$. Limiting $\sigma_{v}$ to be larger than $0.2 \mathrm{~m} \mathrm{~s}^{-1}$ reduces the overestimation to $74 \%$ from $88 \%$. Unexpectedly, the scatter in the concentration estimates $\left(s_{g}=2.07\right)$ is smaller than that corresponding to the use of the observed $\sigma_{v}$ (see Fig. 7, $s_{g}=2.43$ ). The correlation between the concentration estimates and observations is also improved, with $r^{2}=0.54$ compared with $r^{2}=0.50$ in the upper right plot of Fig. 7. These results either indicate that measurements 

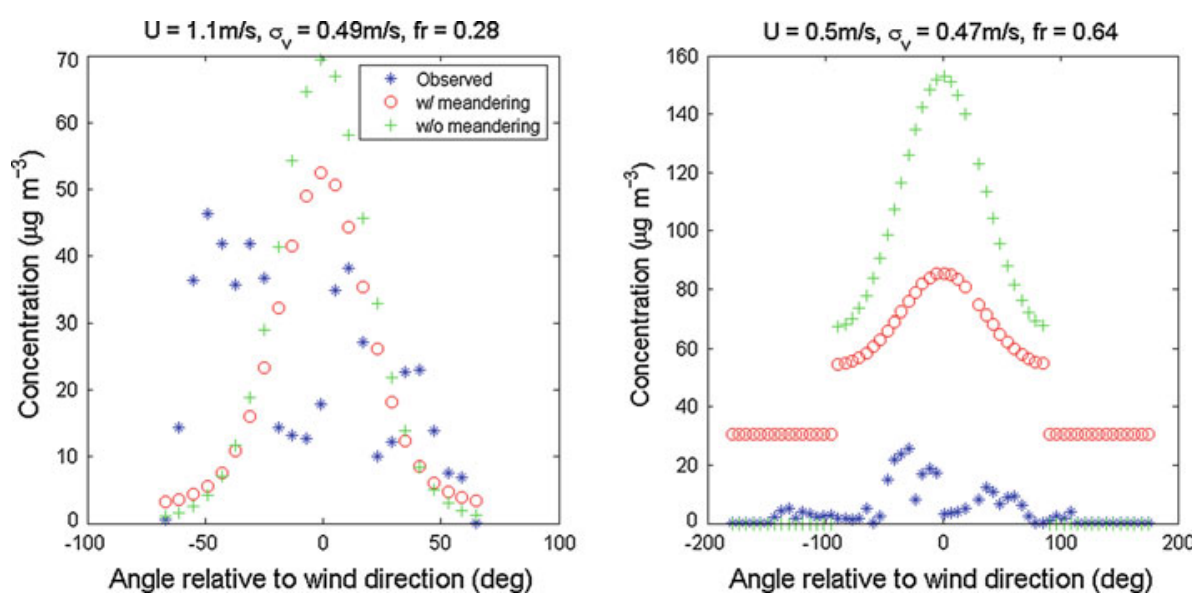

Fig. 8 Variation of concentration with angle relative to the mean wind direction for Test \# 10 (left) and Test \# 8 (right) of the Idaho Falls experiment. Stars represent observations, circles represent model estimates when meandering is considered, and pluses correspond to model estimates when meandering is not considered
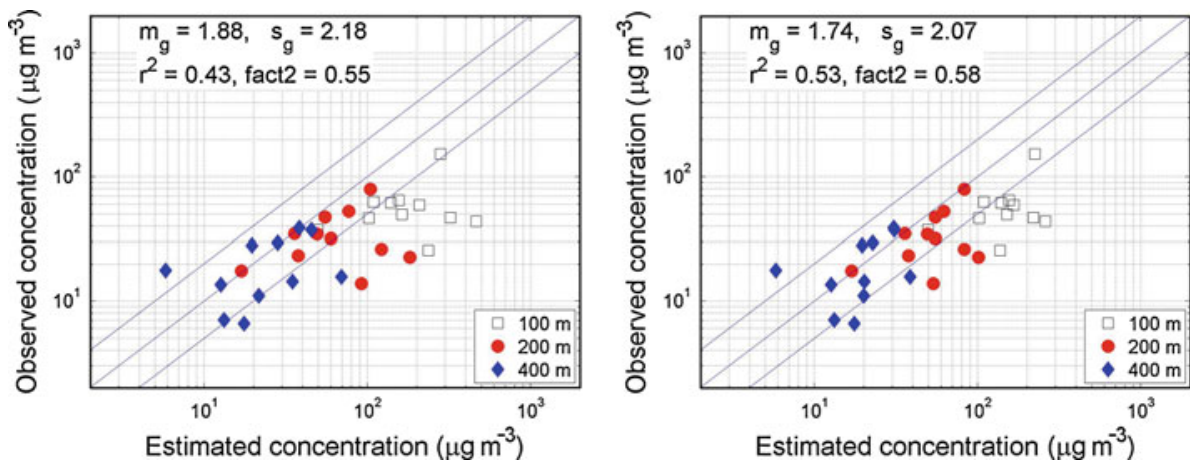

Fig. 9 Comparison of centreline concentrations from the numerical model with observations from the Idaho Falls experiment. Meandering is modelled with Eq. 27. $\sigma_{v}$ is estimated using $\sigma_{v}=1.9 u_{*}$ (left panel), and with a lower limit of $0.2 \mathrm{~m} \mathrm{~s}^{-1}$ (right panel)

of $\sigma_{\theta}$ may not be reliable under low wind speeds, or that a steady-state dispersion model is not applicable under these conditions.

Finally, we examine the performance of the numerical model using several levels of wind measurements. Since the similarity relation, Eq. 8, might not hold during low wind-speed conditions, we calculate the wind speed in Eq. 7 using the power law profile, $U / U_{r}=\left(z / z_{r}\right)^{p}$, where $p$ is obtained by fitting the profile to the observed wind speeds at $1,2,4,8,16 \mathrm{~m}$ of the Prairie Grass experiment. The power, $p$, is obtained from the observed wind speeds at 2, 4, 8, 16,32 , and $61 \mathrm{~m}$ of the Idaho Falls experiment. The advantage of using several levels of wind measurements can be seen by comparing Fig. 10 with the upper left plot of Fig. 6 and the upper right plot of Fig. 7. The overestimation of concentration is reduced using wind speeds fitted to several levels of measurements. However, the correlation coefficients between the estimates and observations are comparable with those when a single level of wind measurement is used: $r^{2}=0.63$ compared with $r^{2}=0.62$ for the Prairie Grass experiment and $r^{2}=0.51$ compared with $r^{2}=0.50$ for the Idaho Falls experiment. 



Fig. 10 Comparison of centreline concentrations from the numerical model with observations from the Prairie Grass experiment (left) and the Idaho Falls experiment (right). The wind speed profile is described with a power law fitted to wind speed measurements at several levels

\section{Conclusions}

We evaluated the performance of two steady-state dispersion models in explaining observations from two tracer studies, the Prairie Grass experiment and the Idaho Falls experiment, under stable low wind-speed conditions. Both these models have been shown to perform well in describing concentrations observed in the Prairie Grass experiment when the wind speeds are above $2 \mathrm{~m} \mathrm{~s}^{-1}$ at a height of $1 \mathrm{~m}$. When applied to low wind-speed conditions, we find that about $50 \%$ of the concentration estimates are within a factor of two of the observations, but the scatter is large: the $95 \%$ confidence interval of the ratio of the observed to estimated concentrations is $\approx 4$. These performance statistics are comparable to those obtained by Sharan and Yadav (1998) using a model that includes along-wind diffusion, which might be important under low wind speeds. This result suggests that ignoring diffusion along the plume in the two models examined herein is empirically justified. It is also consistent with the results of Cirillo and Poli (1992), who showed that the performance of a standard Gaussian model with the horizontal spread estimated from the measured $\sigma_{v}$ differed little from that of a model that considered along-wind diffusion explicitly.

A combination of the two-dimensional diffusion equation and Eckman (1994) model for horizontal dispersion provides a better description of dispersion from near-surface point releases than does AERMOD. This suggests the need to consider the variation of wind speed with height in modelling near-surface dispersion. The performance of both models depends on using measurements of the standard deviation of horizontal turbulent velocity fluctuations, $\sigma_{v}$, to estimate horizontal plume spread. The models overestimate concentrations if $\sigma_{v}$ is estimated from the surface friction velocity. The inclusion in a model of the effects of wind meandering reduces the overestimation of concentrations resulting from the Gaussian plume formulation for horizontal spread. We then examined the estimation of meteorological inputs at low wind speeds, and the impact of using these estimates on modelling the dispersion of near-surface releases. An analysis of data from the Cardington tower indicates that Monin-Obukhov similarity theory generally underestimates the surface friction velocity at low wind speeds. These estimates can be empirically modified to reduce the underestimation, leading to estimates of vertical dispersion that result in improved descriptions of concentrations observed during the Prairie Grass and Idaho Falls experiments under low wind-speed conditions. 
Acknowledgment The research described in this paper was sponsored by the National Science Foundation under grant ATMOS 0430776 and the California Energy Commission.

Open Access This article is distributed under the terms of the Creative Commons Attribution Noncommercial License which permits any noncommercial use, distribution, and reproduction in any medium, provided the original author(s) and source are credited.

\section{References}

Anfossi D, Alessandrini S, Trini Castelli S, Ferrero E, Oettl D (2006) Tracer dispersion simulation in low wind speed conditions with a new 2D Langevin equation system. Atmos Environ 40:7234-7245

Arya SP (1995) Modelling and parameterization of near-source diffusion in weak winds. J Appl Meteorol $34: 1112-1122$

Barad ML (ed) (1958) Project Prairie Grass. A field program in diffusion. Geophysical research paper no. 59, vols I (300 pp) and II (221 pp). AFCRF-TR-58-235. Air Force Cambridge Research Center, Bedford

Brusasca G, Tinarelli G, Anfossi D (1992) Particle model simulation of diffusion in low wind speed stable conditions. Atmos Environ 26A:707-723

Businger JA (1973) Turbulent transfer in the atmospheric surface layer. In: Haugen DH (ed) Workshop on micrometerology. American Meteorological Society, Boston, pp 67-100

Carruthers DJ, Holroyd RJ, Hunt JCR, Weng W-S, Robins AG, Apsley DD, Thompson DJ, Smith FB (1994) UK-ADMS: a new approach to modelling dispersion in the earth's atmospheric boundary layer. J Wind Eng Ind Aerodyn 52:139-153

Caughey SJ, Wyngaard JC, Kaimal JC (1979) Turbulence in the evolving stable boundary layer. J Atmos Sci 36:1041-1052

Chang JC, Hanna SR (2004) Air quality model performance evaluation. Meteorol Atmos Phys 87:167-196

Cimorelli AJ, Perry SG, Venkatram A, Weil JC, Paine RJ, Wilson RB, Lee RF, Peters WD, Brode RW (2005) AERMOD: a dispersion model for industrial source applications. Part I: General model formulation and boundary layer characterization. J Appl Meteorol 44:682-693

Cirillo M, Poli A (1992) An intercomparison of semiempirical diffusion models under low wind speed, stable conditions. Atmos Environ 26A:765-774

Eckman RM (1994) Re-examination of empirically derived formulas for horizontal diffusion from surface sources. Atmos Environ 28:265-272

Gryning SE, Ulden APvan, Larsen S (1983) Dispersion from a ground level source investigated by a K-model. Q J Roy Meteorol Soc 109:355-364

Izumi Y (1971) Kansas 1968 field program data report. Environmental research papers, no. 379, AFCRL-720041. Air Force Cambridge Research Laboratories, Bedford, Massachusetts

Luhar AK (2010) Horizontal turbulence and dispersion in low-wind stable conditions. In: Proceeding of the 13th conference on harmonisation with atmospheric dispersion modelling for regulatory purposes, 1-4 June, Paris, France, pp 863-867

Luhar AK, Hurley PJ, Rayner KN (2009) Modelling near-surface low winds over land under stable conditions: sensitivity tests, flux-gradient relationships, and stability parameters. Boundary-Layer Meteorol 130:249-274

Nieuwstadt FTM, van Ulden AP (1978) A numerical study on the vertical dispersion of passive contaminants from a continuous source in the atmospheric surface layer. Atmos Environ 12:2119-2124

Oettl D, Almbauer RA, Sturm PJ (2001) A new method to estimate diffusion in stable, low wind conditions. J Appl Meteorol 40:259-268

Panofsky HA, Dutton JA (1984) Atmospheric turbulence. Wiley, New York, 397 pp

Sagendorf JF, Dickson CR (1974) Diffusion under low wind speed, inversion conditions. NOAA Technical Memorandum ERL ARL-52, 89 pp

Sharan M, Yadav AK (1998) Simulation of experiments under light wind, stable conditions by a variable K-theory model. Atmos Environ 32:3481-3492

Sharan M, Yadav AK, Singh MP (1995) Comparison of sigma schemes for air pollutant dispersion in low winds. Atmos Environ 29:2051-2059

van Ulden AP (1978) Simple estimates for vertical dispersion from sources near the ground. Atmos Environ 12:2125-2129

Venkatram A (1980) Estimating the Monin-Obukhov length in the stable boundary layer for dispersion calculations. Boundary-Layer Meteorol 19:481-485 
Venkatram A (1992) Vertical dispersion of ground-level releases in the surface boundary layer. Atmos Environ 26A:947-949

Venkatram A (2004) The role of meteorological inputs in estimating dispersion from surface releases. Atmos Environ 28:2439-2446

Venkatram A (2008) Computing and displaying model performance statistics. Atmos Environ 24:6862-6868

Venkatram A, Princevac M (2008) Using measurements in urban areas to estimate turbulent velocities for modeling dispersion. Atmos Environ 42:3833-3841

Venkatram A, Isakov V, Yuan J, Pankratz D (2004) Modeling dispersion at distances of meters from urban sources. Atmos Environ 38:4633-4641 